\title{
Pressure Bifurcation Phenomenon on Supersonic Blowing Trailing Edges
}

\author{
Alejandro Martinez-Cava, ${ }^{*}$ Yinzhu Wang, ${ }^{\dagger}$ Javier de Vicente, ${ }^{\ddagger}$ and Eusebio Valero ${ }^{8}$ \\ Technical University of Madrid, E-28040 Madrid, Spain
}

DOI: $10.2514 / 1 . J 057056$

\begin{abstract}
Turbine blades operating in transonic-supersonic regime develop a complex shock wave system at the trailing edge: a phenomenon that leads to unfavorable pressure perturbations downstream and can interact with other turbine stages. Understanding the fluid behavior of the area adjacent to the trailing edge is essential in order to determine the parameters that have influence on these pressure fluctuations. Colderflow, bled from the high-pressure compressor, is often purged at the trailing edge to cool the thin blade edges, affecting the flow behavior and modulating the intensity and angle of the shock waves system. However, this purge flow can sometimes generate nonsymmetrical configurations due to a pressure difference that is provoked by the injected flow. In this work, a combination of Reynolds-averaged Navier-Stokes simulations and a global stability analysis is employed to explain the physical reasons of this flow bifurcation. By analyzing the features that naturally appear in the flow and becone dominant for some value of the parameters involved in the problem, an antisymmetrical global mode, which is related to the sudden geometrical expansion of the trailing-edge slot, is identified as the main mechanism that forces the changes in the flow topology.
\end{abstract}

\section{Nomenclature}

$d$

linearized Jacobian matrix

volume matrix

$=$ trailing edge thickness

$=$ flux tensor

$=$ frequency

$=$ turbulent kinetic energy

number of grid points

number of faces on subdomain

number of fluid variables

normal direction to the body surface

specific dissipation rate of $k$

$=$ projection operator matrix

pressure measured at the base region

- pressure measured at the base region when no purge flow is applied base flow vector field

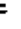

discretized vector state solution in subdomain $\Omega_{i}$

vector of small perturbations

vector of complex disturbances

residual vector in subdomain $\Omega_{i}$

global density residual

Strouhal number

\section{temperature}

time

velocities in $x, y$, and $z$ directions, respectively

vector of coordinate directions $(x, y, z)$

dimensionless wall-normal distance

Received 15 January 2018; revision received 29 June 2018; accepted for publication 2 October 2018: published online 5 December 2018. Copyright (c) 2018 by Universidad Politécnica de Madrid (UPM). Published by the American Institute of Aeronautics and Astronautics, Inc., with permission. All requests for copying and permission to reprint should be submitted to $\mathrm{CCC}$ at www.copyright.com; employ the ISSN 0001-1452 (print) or 1533-385X (online) to initiate your request. See also AIAA Rights and Permissions www,aias.org/randp.

*Early Stage Researcher, School of Aeronautical and Space Engineering. Plaza Cardenal Cisneros 3; alejandro.martinezcava@upm.es.

${ }^{t}$ Early Stage Researcher, School of Aeronautical and Space Engineering. Plaza Cardenal Cisneros 3: y, wang@upm.es.

${ }^{\ddagger}$ Associate Professor of Applied Mathematics, School of Aeronautical and Space Engineering. Plaza Cardenal Cisneros 3; $\mathbf{j}$.devicente@upm.es.

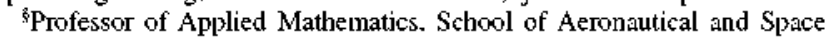
Engineering, Plaza Cardenal Cisneros 3; ensebio.valero@upm.es.

$\begin{array}{ll}z & =\text { spanwise direction } \\ \beta & =\text { spanwise wave number } \\ \rho_{f} & =\text { density measured at the base region } \\ \rho_{\text {purge }} & =\text { density measured at the cavity plenum } \\ \Omega & =\text { control volume } \\ \Omega_{i} & =\text { mesh subdomain } \\ \omega & =\text { complex eigenvalue } \\ \omega_{i} & =\text { eigenvalue imaginary part (pulsation) } \\ \omega_{r} & =\text { eigenvalue real part (amplification rate) }\end{array}$

\section{Introduction}

A IR transport has experienced a very large growth in the last few decades, with a significant socioeconomic relevance for integration and development at regional and intemational levels. From a technological point of view, the aerospace industry is constantly at the cutting edge of scientific development. Continuous improvements in aerodynamics, material sciences, manufacturing processes, avionics, control, and navigation systems make aircraft more efficient and safer every day. In particular, aircraft engines tend toward more compact architectures, the structural safety limits are tighter, and aerostructural couplings may lead to low- and high-cycle fatigue issues. Such aerostructural interactions are significantly detrimental when the intemal flow velocities approach the speed of sound.

Turbomachinery airfoils can operate in the transonic or supersonic flow regime, experiencing complex shock wave systems at the trailing edge that can lead to unfavorable pressure perturbations affecting the life span of the turbine components. Part of the aerostructural coupling has its origin on the flow fluctuations that take place at the area adjacent to the trailing edge. known as the base region. The evaluation of the parameters driving the compression and expansion waves, shear layers, and vortex shedding should then allow us to mitigate their unsteady couplings with the turbine blades.

Figure 1 summarizes the flow topology of a supersonic trailing edge, which is often approximated by a simplified model based on the supersonic flow over a flat plate end. The upstream boundary layer remains attached at the trailing-edge comer, and it separates immediately downstream. developing a shear layer while the main flow accelerates through a Prandtl-Meyer expansion fan. The upper and lower shear layers propagate downstream and eventually merge at the wake on a point of confluence, creating a dead air zone limited by both shear layers and the blunt trailing edge, namely, the base region. This zone is characterized by low momentum and constant pressure, having a strong influence on several features of the surrounding flowfield, such as the vortex oscillation intensity or 


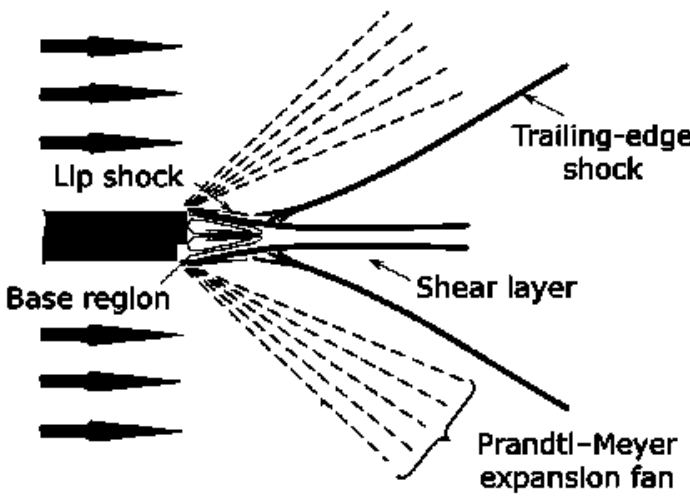

Fig. 1 Schematic representation of supersonic flow on a blunt trailing edge. Image modified from the work of Saracoglu et al. [15].

shock wave position and angle. At the confluence point, the flow changes its direction notably, being forced to compress through a system of strong trailing-edge shocks. The degree of compression, and hence the strength of the shock waves, highly depends on the base region properties. Furthermore, a weak compression wave, called separation or lip shock, is formed at the point of flow detachment in order to adapt the pressure gradient between the shearing and the downstream flow. The base region has been an object of study for a long time [1-4], and its influence and characteristic topology on turbine cascades $[5,6]$ or the supersonic turbulent wake of axisymmetric bodies [7-9] has been repeatedly revisited.

The thin trailing edge of turbine blades cannot be protected using internal cooling passages, and so cooler flow bled from the highpressure compressor is purged through slots or orifices to avoid the melting of the turbine components. As shown by several authors [10,11], this cooling could be exploited to modulate the base region properties. Different studies [12-14] have been devoted to examining the effects of trailing-edge bleeding on the base region. Of particular importance, Saracoglu et al. [15] investigated the flow topology at the blunt trailing edge as a function of the cooling flow intensity, showing the dependence of the base pressure with the blowing magnitude. A relevant result of the study was the presence of nonsymmetric configurations at the base flow (shock intensity and angle of the shock wave) for a specific range of blowing rates (Fig. 2). Two different bifurcations from symmetric to nonsymmetric configurations were observed: the first one arising when the base pressure was close to reaching its maximum value; and the second one appearing for higher blowing ratios, close to a local minimum value for the base pressure.

The objective of the present research is to combine Reynoldsaveraged Navier-Stokes (RANS) simulations and a linear stability theory to explain the mechanisms that generate the described nonsymmetric configuration. Only the first pressure bifurcation is analyzed in detail, focusing on the area in which the purge flow provokes an important increment on the base pressure, reducing the intensity of the shock waves and the base pressure losses. A thorough analysis of the flow topology of a supersonic blowing trailing edge is carried, focusing on the recirculation areas of the base region and showing how those areas interact, generating changes on the injected

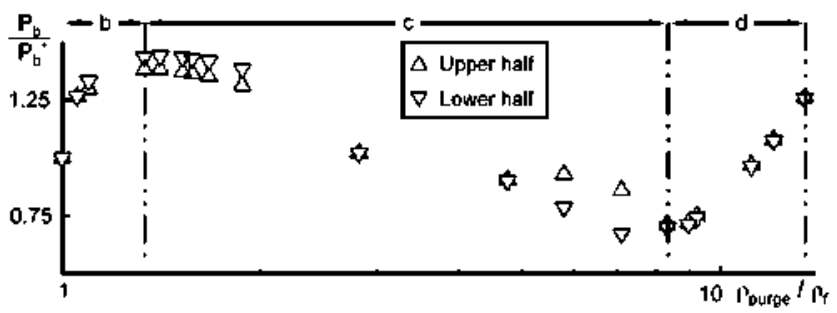

Fig. 2 Base pressure correlation at the trailing edge as a function of the density ratio of the bleeding rate. Image reprinted from the work of Saracoglu et al. [15]. flow, and therefore in the complex wave system. Those changes are then also identified using a global stability analysis. The stability analysis studies the growth or decay of perturbations superimposed upon a steady base flow, and it has proven to be effective in the analysis of incompressible and compressible flows: either in laminar or turbulent configurations. The analysis aims to identify which features can naturally appear in the flow and become dominant for some particular value of the parameters. Depending on the nature of the phenomenon, the analysis can be performed over a steady base flow solution [16-18], over an averaged mean flow [19-21], or using timestepping or Dynamic Mode Decomposition [22] techniques for the study of transient phenonmen [23,24]. In this work, steady base flows were considered to characterize the stability behavior of the system. The results of this study shed some light on the pressure bifurcation detected by Saracoglu et al. [15], identifying the physical global mode responsible of the nonsymmetrical conditions as a function of the cooling flow purge intensity. We anticipate that the results of the stability analysis match the bifurcation predicted by RANS simulations, adding extra information about the underlying physical mechanisms. The unstable configuration seems to be related to the sudden geometrical expansion of the flow at the end of the cooling slot. The associated instability forces the purge flow direction to deflect and impact one of the shear layers, generating an additional shock wave system. Such changes in the vicinity of the trailing edge would have a great effect on the loads affecting the adjacent blades and turbine stages. The identified global mode changes its shape with the blowing rate intensity, as does the flow topology of the base region, giving the stability analysis valuable information about where the perturbations are located.

The rest of the paper is organized as follows. Section $I$ gives an overview the mathematical formulation of the stability analysis and implementation details, opening the door to future applications in flow control. Section III summarizes the numerical procedures to obtain the base flow and stability solutions. In Sec. IV, the main results are described, and we finish in Sec. $V$ with the main conclusions of this study.

\section{Mathematical Model}

\section{A. Flow Solver}

The compressible version of the Reynolds-averaged NavierStokes equations is used to model the flow, closing the mathematical system with the Wilcox $k-w$ turbulence model. This set of equations can be written in conservative form as follows:

$$
\frac{\partial}{\partial t} \int_{\Omega} q \mathrm{~d} \Omega=-\int_{\partial \Omega} \overline{\bar{F}} \cdot n \mathrm{~d} S
$$

where vector $q$ comprises the conservative variables (density, momentum, and energy) and turbulent quantities, whereas $\Omega$ is a control volume with the boundary $\partial \Omega$ and outer normal $n$. The specific heat capacities of the gas at constant volume and pressure are both assumed constant, and so, consequently, it can be defined as the constant adiabatic coefficient of the ideal gas. A finite volume approach is selected for the spatial discretization; namely, the change of the flow conditions in a control volume $\Omega$ is given by the normal component of the flux through the control volume boundary $\partial \Omega$. The flux density tensor $\overline{\bar{F}}$ can be decomposed along the three Cartesian coordinate directions and comprises the inviscid, viscous, and turbulent fluxes. Details of the RANS formulation can be found elsewhere and, for the particular case of the $k-w$ turbulence model, in the work of Wilcox [25].

The finite volume DLR TAU code [26] is used to obtain a steadystate solution of the base flow equations. The flow domain $\Omega$ is discretized using a dual mesh into a finite number of subdonains $\Omega_{i}$, $i=1 \ldots N$, where each subdomain contains $\mathcal{N}_{f}$ faces. This approach is well suited to unstructured grids, using an edge-based data structure to optimize the memory requirements and the

\footnotetext{
"Bold variables are used to refer vectors.
} 
computational performance of the solver. The time-accurate threedimensional Navier-Stokes equations are marched in time toward steady state by a backward Euler implicit scheme, which is solved approximately by a lower-upper symmetric Gauss-Seidel method iterations procedure. Local time stepping and multigrid algorithms are adopted to accelerate convergence, allowing us to converge a forced steady solution of the RANS equations from flow configurations that would otherwise develop an unsteady behavior if run with an unsteady solver.

Thus, following the method of lines, the spatial discretization of system (1) gives rise to a system of ordinary differential equations, which can be written in general form for a subdomain $\Omega_{i}$ as follows:

$$
|\Omega|_{i} \frac{\partial \boldsymbol{q}_{i}}{\partial t}+\boldsymbol{R}_{i}=\mathbf{0} . \quad \boldsymbol{R}_{i}=\sum_{j=1}^{N_{j}} \overline{\bar{F}}_{j} \boldsymbol{n}_{j}, \quad i=1 \ldots N
$$

where $\boldsymbol{R}_{i}$ is the residual in subdomain $\Omega_{i}$, which is equivalent to the flux contributions to this subdomain; and $q_{i}$ represents a discretized vector state solution in subdomain $\Omega_{i}$. Vectors $\boldsymbol{q}_{i}$ and $\boldsymbol{R}_{i}$ have dimensions that depend on the number of fluid variables $N_{v}$ considered. Namely, $N_{v}=4,5,6$, or 7 , depending whether the problem is laminar or turbulent, or two- or three-dimensional. The number of subdomains depends on the number of elements of the computational mesh.

Likewise, nonslip boundary conditions and an adiabatic wall are imposed on the body surface as follows:

$$
u=v=w=0 \quad \frac{\partial T}{\partial n}=0
$$

where $n$ is the normal direction to the body surface, and $T$ stands for the temperature. At the external boundaries, a far-field boundary condition is used. The convective fluxes crossing the far-field boundary faces are calculated using the advection upstream splitting method (known as AUSM) Riemann solver [27], and the flow conditions outside the boundary faces are determined by employing the Whitfield theory [28]. The freestream turbulence intensity on the far-field boundaries is set to $0.1 \%$, and the freestream ratio of the eddy to laminar viscosity is equal to $\mu_{t \infty} / \mu_{\infty}=0.001$.

\section{B. Stability Analysis}

The stability analysis studies the growth or decay of perturbations superimposed on to a usually steady solution of the Navier-Stokes (NS) equations. The analysis can identify which particular features are prone to evolve under modifications of the flow conditions, either by introducing a perturbation or caused by a modification of some physical or geometrical parameter. The growth of these features will give rise to a completely different flow configuration. Examples of the application of flow stability to fluid dynamics problems can be found in the literature for a large variety of flow topologies [29-34].

Following the linear stability theory, any flow quantity can be decomposed into a steady or time-periodic component; upon which, small perturbations are allowed to develop. We can express, without loss of generality, that

$$
q(x, t)=Q(x)+\varepsilon \tilde{q}(x, t)
$$

where $\varepsilon \ll 1$, and $Q$ is the so-called base flow, which is the point of equilibrium of $\mathrm{Eq}$. (2). The small perturbations are modeled as the term $\tilde{q}(x, t)$.

This decomposition is introduced into the NS equations [Eq. (2)], and a Taylor series expansion is performed around the point of equilibrium $Q$. Neglecting terms of order $\varepsilon^{2}$ and assembling all the unknowns in vector/matrix notation, the new system in perturbed variables is obtained:

$$
\varepsilon B \frac{\partial \tilde{q}}{\partial t}=\boldsymbol{R}(Q+\varepsilon \tilde{q}) \approx \boldsymbol{R}(Q)+\varepsilon\left[\frac{\partial \boldsymbol{R}}{\partial \boldsymbol{q}}\right]_{Q} \tilde{\boldsymbol{q}}
$$

where the diagonal matrix $B$, with leading dimension $N_{v} \times N$, contains the volumes associated to each cell; and $[\partial R / \partial q]_{Q}$ represents the Jacobian of the fluxes evaluated in the base flow. The stability of the system is therefore governed by the properties of the last. If the base flow $Q$ is steady, linear system (5) is autonomous and its coefficients are independent of $t$.

If a Fourier expansion is considered in time $t$ and the spanwise $z$ direction, the following decomposition holds:

$$
\tilde{\boldsymbol{q}}(\boldsymbol{x}, t)=\hat{\boldsymbol{q}}(x, y) e^{i(\beta z-\omega t)}+\text { c.c. }
$$

where $\omega$ is a complex scalar, $\beta$ represents the spanwise wave number, $\hat{q}$ describes the complex disturbance function of $x$ and $y$, and c.c. stands for the complex conjugate. Complex conjugation is included because the pair $(\hat{q}(x, y), \omega)$ and its complex conjugate are both solutions of the linearized NS equations, whereas $\tilde{q}$ is real.

In pure two-dimensional cases, such as the one analyzed here, the velocity component and all derivatives in the spanwise direction are neglected for both the base flow and disturbances, and $\beta$ is set to zero. Renaming $-i \omega \rightarrow \omega$, the final decomposition is finally considered:

$$
\tilde{q}(x, t)=\hat{q}(x, y) e^{\omega t}+\mathrm{c} \cdot \mathrm{c} .
$$

Substitution of the ansatz given by Eq. (7) into linear system (5) and considering $\boldsymbol{R}(\boldsymbol{Q})=0$ (obtained by the steady RANS computation), Eq. (5) is transformed into a two-dimensional generalized eigenvalue problem:

$$
\left[\frac{\partial R}{\partial \boldsymbol{q}}\right]_{Q} \hat{\boldsymbol{q}}=\omega B \hat{\boldsymbol{q}}
$$

which can also be expressed as follows:

$$
A \hat{q}=\omega B \hat{q}
$$

with

$$
A=\left[\frac{\partial R}{\partial \boldsymbol{q}}\right]_{Q}
$$

being the linearized Jacobian matrix, and $\omega=\omega_{r}+i \omega_{i}$ as the complex eigenvalues of the generalized system. According to this formulation, the real part $\omega_{r}$ is referred as the amplification rate of the corresponding eigenmode, with the imaginary part $\omega_{i}$ being the pulsation. which is related to the associated frequency as $S t=\omega_{i} / 2 \pi$. If the real part of the eigenvalue takes a positive value, the associated eigenmode will eventually become dominant over the steady base flow, whereas a negative real part will indicate a damping behavior of the associated eigenmode.

The DLR TAU code includes a linear frequency domain solver, which uses an analytic linearized Jacobjan matrix. This capability notably simplifies the computational effort to obtain the input needed for the stability analysis.

\section{A. Base Flow Solver}

\section{Numerical Procedure}

The domain of reference of the problem (see Fig. 3) had been previously studied by Saracoglu et al. [15] and Gorbachovaet al. [35]. The former used an extensive unsteady RANS (URANS) simulation to give a comprehensive analysis of the effects of the trailing-edge purge flow on the flow topology, and the latter did a first approach to the problem throughout a global stability analysis. The geometrical approximation was a simplification of the industrial problem, in which only the details of the flow in the vicinity of the trailing edge of a turbine blade were studied. The geometry also included the internal cooling passages (which were composed of a cavity plenum in which the purge flow was ejected) and the injector pipe. directly communicating the plenum with the base region.

The computational domain was limited by straight boundaries, with a length of $21.75 \times d$ (with $d$ as the trailing-edge thickness. 


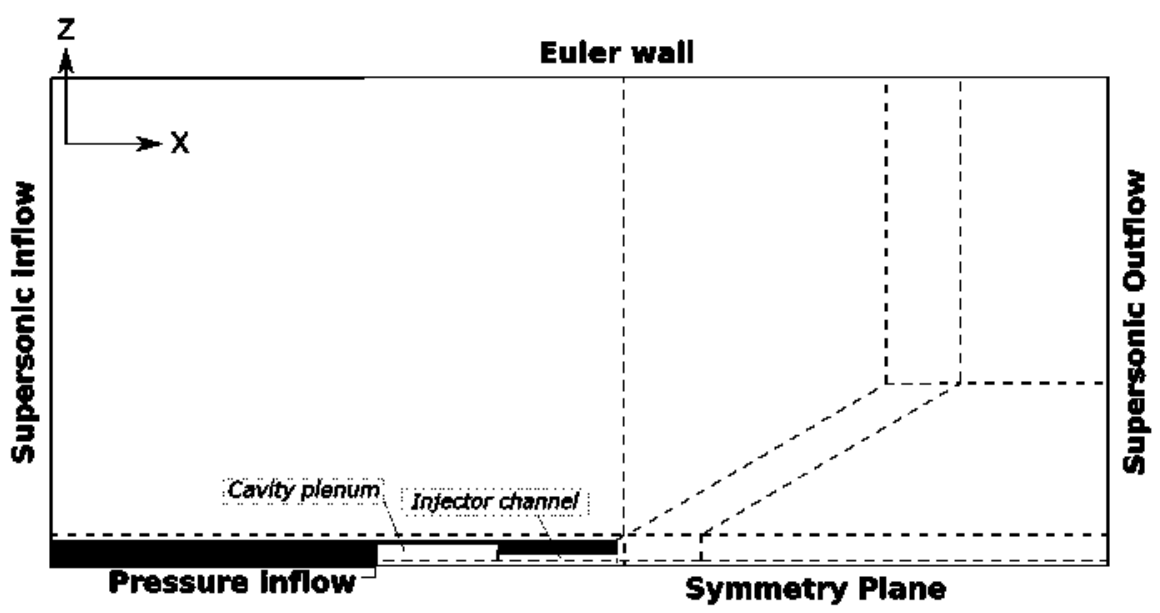

Fig. 3 Geometry definition (solid line) and grid topology (dashed lines) used on the mesh generation. Only the half-domain is shown for simplification.

which was equal to $0.02 \mathrm{~m}$ ) and a width of $20 \times d$. The injector pipe had a length and width of $2.5 \times d$ and $0.3 \times d$, respectively. To keep subsonic conditions inside the cavity plenum, its width was kept three times larger than the injector pipe. The characteristic geometrical lengths of the analysis are shown in Table 1 .

Boundary conditions of the control volume were defined as follows: supersonic inflow and output; the Euler wall and symmetry plane set at the left, right, upper, and lower limits, respectively; the nonslip wall used for all intemal surfaces; and a reservoir-pressure inflow boundary condition set to simulate the input of the cooling in the cavity plenum area (Fig. 3).

The Mach number at the inflow boundary was set to 1.5 , keeping a total temperature of $365 \mathrm{~K}$ and a Reynolds number of $9.4 \times 10^{6}$, based on the trailing-edge length $(11.65 \times d)$. The flow rate at the cooling flow exit (from now on referred to as the blowing rate) was defined by the cooling flow static temperature (kept to $300 \mathrm{~K}$ ) and its total pressure, which was expressed as a percentage of the freestream flow total pressure.

Because the study aimed to understand the different flow features present in the flow and, in particular, the nature of the nonsymmetry behavior, a global stability analysis over the steady symmetric base flow was performed. Thus, only half of the domain was simulated using a RANS approach, imposing a symmetry boundary condition on the plane of symmetry to obtain a steady solution. After obtaining a "half-"base flow, the mesh and the solution were mirrored into the symmetry plane and the stability analysis of the two-dimensional full domain was carried out. The global analysis of the mirrored-full domain around this symmetric flow at different blowing ratios would show nonsymmetric modes in the analysis, which could eventually grow and generate a new nonsymmetric flow configuration. To clarify the exposition, when the term half-domain is used in this text, it will refer to the mesh of Fig. 4, with a symmetry plane on its lower boundary; on the contrary, when the term full domain is used, it will refer to simulations done on a mirrored mesh, and considering upper and lower surfaces of the trailing edge. A similar methodology on the detection of flow bifurcation can be found in the literature on the detection of global modes of symmetric sudden expansions [36] or $\mathrm{X}$-junction flow configurations [24].

Table 1 Fluid domain dimensions

\begin{tabular}{lc}
\hline \hline Boundary & Length, $\mathrm{m}$ \\
\hline Domain length & 0.4346 \\
Domain height & 0.4 \\
Trailing-edge length & 0.233 \\
Trailing-edge height & 0.02 \\
Cavity plenum length & 0.05 \\
Cavity plenum height & 0.018 \\
Injector channel length & 0.05 \\
Injector channel beight & 0.006 \\
\hline \hline
\end{tabular}

The domain was discretized using a quadstructured mesh, which was generated using an $\mathrm{O}$-grid topology and dividing the geometry into regions of interest. Wall-normal grid distances were set small enough so that values of $y^{+}$lower than one were kept on the body surfaces, and all the scales of the boundary layer were fully resolved without the use of any wall model. Grid topology and mesh details are pictured in Figs. 3 and 4. The mesh spatial resolution was concentrated at the contraction-expansion and on the shock wave areas.

To ensure mesh-independent results, a mesh convergence study was performed for a blowing rate of $18 \%$ for a half-domain configuration. Six different meshes of increasing numbers of elements were defined, and they are numbered from M1 to M6. A systematic refinement was performed to evaluate the flow sensitivity to the mesh density. At each step, the mesh was refined by increasing $n$ times the number of nodes, taking mesh $\mathrm{Ml}$ as the reference. This refinement law preserved the topology of the mesh and guaranteed that Richardson extrapolation formulas were applicable to obtain an accurate rate of convergence [37]. The convergence criteria, evaluated through the global density residual res $s_{p}^{\prime \prime}$, was set up to values lower than $10^{-6}$. It was defined as follows:

$$
\left\|\operatorname{res}_{\mu}^{n}\right\|=\sqrt{\sum_{j=1}^{N} \frac{\left[\operatorname{res}_{p}^{n}(j)\right]^{2}}{N}}
$$

where $N$ denoted the number of grid points.

Detailed results from the mesh convergence study are shown in Table 2. At each mesh, the pressure and density values at the trailing edge were monitored. According to these results, mesh M4 was selected as a good compromise between accuracy and computational efficiency.

\section{B. Stability Analysis}

The real nonsymmetric operator $A[\mathrm{Eq}$. (8)] was extracted using the first-discretize-then-linearize technique implemented in the DLR TAU solver, and it was saved using a compressed sparse row format. For the particular case of mesh M4, the leading dimension of matrix $A$ was approximately $1.2 \times 10^{6}$, and the number of nonzero elements was $9.6 \times 10^{7}$. The real diagonal operator $B$, representing the volume of each element, was also obtained from the solver.

For each eigenvalue, there is an associate eigenvector that represents the underlying physical feature [density, momentum in the spatial directions $(x, y, z)$, energy, and turbulent viscosity], which will eventually decay or grow, depending on the sign of the real part of the eigenvalue. To calculate the eigenvalues and eigenvectors of system 9, the implicitly restarted Arnoldi method (IRAM) algorithm implemented in the ARnoldi PACKage library [38] is used. The Annoldi algorithm is a good technique for approximating the eigenvalues of large sparse matrices; however, only the eigenvalues with larger modules can be obtained by a straightforward application of the algorithm. To capture the region of the complex plane in which the unstable eigenvalues lay, a shift-and-invert preconditioning 


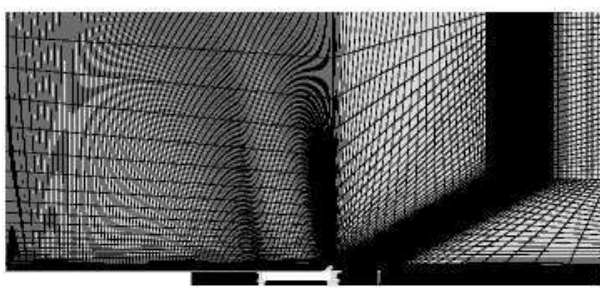

a) Computational mesh

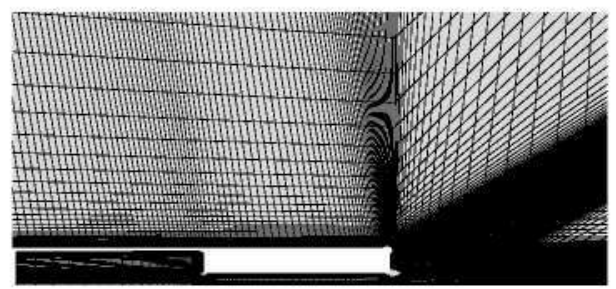

b) Details of the mesh in the cavity and channel area

Fig. 4 Computational mesh. Only one-quarter of the total nodes is represented for clarity.

technique [39] is used. Not all the eigenvalues are computed, but only a finite number close to an area of interest, which is defined by the shift parameter. At each iteration of the IRAM algorithm, the shiftand-invert method requires the inversion of a linear system, which makes this procedure computationally expensive. Moreover, due to the stiffness associated to the underlying compressible and turbulent equation system, Jacobian matrix $A$ is very badly conditioned, and so iterative methods to solve the linear system fail to reach a proper convergence. In this work, a full lower-upper (LU) factorization is performed for Jacobian matrix $A$. This strategy, used here in a compressible finite volume context, has also been followed in the stability analysis of incompressible flows in finite element discretizations and in the context of spectral methods [40-42] and highly compressible flows with turbulence modeling [16]. Unfortunately, the full LU decomposition scales as the cube of the number of unknowns $\left[\left(N_{v} \times N\right)^{3}\right]$, becoming the bottleneck of the overall process and consuming a large amount of computational resources [16]. To partially alleviate this requirement, a sparse matrix format and parallel algorithms are used. This is done through an Open Multi-Processing and Message Passing Interface implementation of the algorithms and the use of the MUltifrontal Massively Parallel sparse Direct Solver library [43].

Additionally, in order to reduce the dimension of the eigenvalue problem, a domain reduction technique was applied on this work before the LU decomposition.

\section{Domain Reduction Methodology}

For completeness of exposition, we shortly reproduce here the mathematical foundation of this method; additional information can be found in the work of Sanvido et al. [44].

The Arnoldi method belongs to the broad category of projection methods that aims to obtain the part of the spectrum of matrix $A$ where the dominant eigenpairs lay.

The domain reduction (DR) strategy can be considered, somehow, a projection technique in which the original matrix is reduced to a computationally affordable one. The projection in the domain reduction procedure is a purely geometrical technique; starting from a domain of reference $\Omega_{n}$ (defined from the computational mesh) and a vector of unknowns $\boldsymbol{q}_{n}$ (comprising the variables involved in the Linear Navier-Stokes equations), we consider a subdomain of the computational mesh $\Omega_{m}$, which is a region of interest that contains $m<n$ degrees of freedom. For simplicity, we order the unknowns

Table 2 Mesh convergence analysis performed for a blowing rate of $18 \%$ for a half-domain configuration ${ }^{\mathrm{a}}$

\begin{tabular}{lccc}
\hline \hline Mesh & Number of nodes & $P_{b} / P_{b}^{*}$ & $\rho_{\text {purge }} / \rho_{f}$ \\
\hline M1 & 12,010 & 1.26932202 & 1.03157676 \\
M2 & 48,260 & 1.29883128 & 1.03038449 \\
M3 & 104,630 & 1.30545566 & 1.02675552 \\
M4 & 207,106 & 1.31826117 & 1.01727924 \\
M5 & 321,090 & 1.31929642 & 1.01407829 \\
M6 & 424,640 & 1.31858961 & 1.01426252 \\
\hline \hline
\end{tabular}

${ }^{n} P_{b} / P_{b}^{*}$ represent the relation between the pressure with and without purge flow applied, and $\rho_{\text {purgo }} / \rho_{f}$ is the relation between the density values at the cavity plenum and at the base region. related to the subdomain in the first positions and in the global vector of unknowns $\boldsymbol{q}_{n}$. Namely,

$$
\boldsymbol{q}_{n}=\left\{q_{1} \cdots q_{n}\right\}^{T}=\left\{q_{\{1 \cdots m\}} \mid q_{\{(m+1) \cdots n\}}\right\}^{T}
$$

Under this assumption, the projection of the unknowns from $\Omega_{n}$ to $\Omega_{m}$ is easily performed through the projection operator $P$ defined as follows:

$$
\boldsymbol{q}_{m}=P \boldsymbol{q}_{n} \quad \text { with } P=\left(\begin{array}{ccccc}
1 & 0 & 0 & \ldots & 0 \\
0 & 1 & 0 & \ldots & 0 \\
0 & \vdots & \vdots & \ddots & 1 \\
0 & 0 & \ldots & 0 & 0 \\
0 & 0 & \ldots & 0 & 0
\end{array}\right)_{(n, m)}
$$

where the first $m$ rows of $P$ form an identity matrix of order $m$, and the remaining $(n-m)$ rows are identically zero. It is easily checked that $P$ is a orthonormal projector and its columns form a orthonormal basis on the subdomain defined by $\Omega_{m}$, and so the projection of the Jacobian $A_{n}$ onto this subspace is easily computed as follows:

$$
A_{m}=P^{T} A_{n} P
$$

where the superscript $T$ stands for the transpose, and $A_{m}$ is the orthonormal projection of $A_{n}$ in the subspace of dimension $m$.

Hence, the original Jacobian (in $\Omega_{n}$ ) can be replaced by its projection (in $\Omega_{m}$ ) and the "reduced" eigenpairs computed. Sanvido et al. [44] showed that the DR technique permits the recovery of the most relevant disturbances related to the specific region of interest, with the added value of filtering part of the spectra that is not relevant for that particular region. The authors proved that the approximation would be valid as long as the reduced domain contained the structural sensitivity region of the dominant eigenmodes.

\section{Domain Reduction Numerical Validation}

On the case of study, the region of interest was concentrated around the base region and the cavity-injector area (Fig. 5b), representing a small section of the whole computational domain.

To illustrate the influence of the domain used on the stability analysis, part of the eigenspectrum for a blowing rate of $18 \%$ is shown in Fig. 5a. The abscissa represents the number of requested eigenvalues, set up as one-third of the projected Krylov subspace size [Number of Columns of V (NCV)]; and the ordinate shows the real part of the least stable eigenvalue $\omega_{r}$. Three different domains, denoted as DR1 to DR3, are considered and compared to the results obtained without any domain reduction technique (Fig. 5b). The tendencies of the results are in good agreement with the one predicted by Sanvido et al. [44], in which the eigenvalue showed a strong sensitivity to the domain extension. It is not the aim of this work to explain the reason for these differences but only the applicability of the methodology. A complete description of the sources and the estimation of the errors was given by Sanvido et al. [44]. According to these results, DR3 was used for the stability computations on the rest of this work.

The number of degrees of freedom of the reduced domain DR3 was 436,320 , which meant a total of $\simeq 10^{11}$ elements of the Jacobian matrix, and a number of nonzero elements of approximately $3.3 \times 10^{7}$, which was almost one-third of the nonzero 


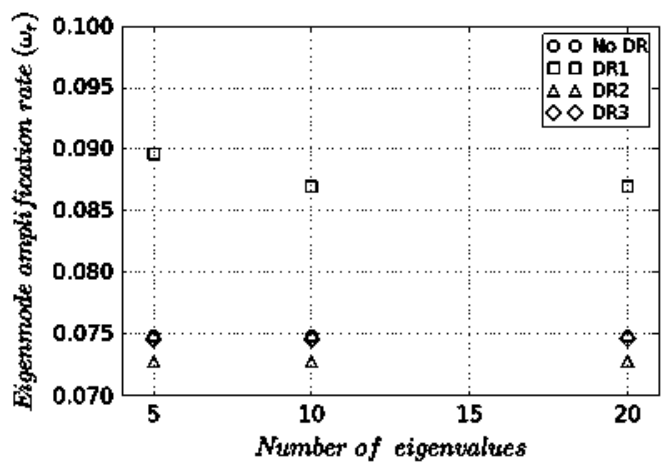

a) Influence of the domain reduction technique on the eigenvalue analysis

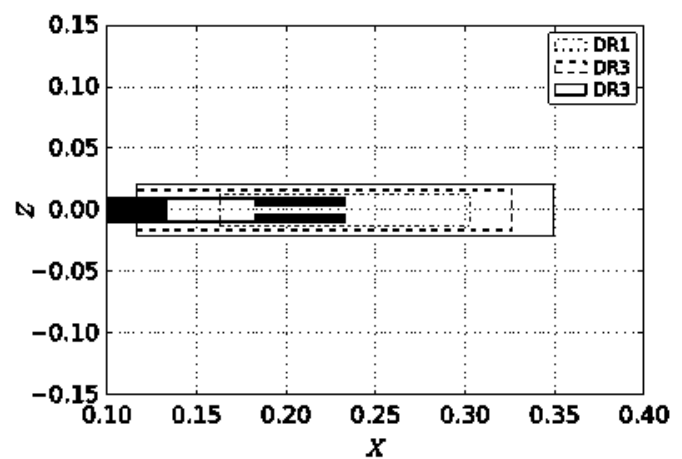

b) Illustration of the different reduced donains used for the analysis

Fig. 5 Domain reduction technicjue convergence analysis.

elements of the original matrix. To highlight the advantages of the use of a reduced domain, statistics calculated on a local workstation equipped with eight IntelCore ${ }^{\mathrm{TM}}$ i7-6700K CPUs with $32 \mathrm{~GB}$ of available RAM were collected. Before the application of the DR technique, the solve of the eigenvalue problem would take a total of $700 \mathrm{~s}$ to finalize, consuming more than $25 \mathrm{~GB}$ of RAM and with a number of nonzero elements of $8.7 \times 10^{8}$ for the factorized matrix. After the application of the domain reduction, the time to solve the eigenvalue problem was reduced to $210 \mathrm{~s}$, demanding only $8 \mathrm{~GB}$ of RAM and with a number of nonzero elements of $2.8 \times 10^{8}$ for the factorized matrix.

\section{Results}

\section{A. Flow Topology Analysis}

The results shown here are expressed as a function of the blowing rate intensity, defined as mentioned by its static temperature and total pressure, with the last defined as a percentage of the freestream total pressure. Due to its widespread use in the literature related to turbomachinery trailing-edge cooling, the freestream-to-purge density ratio $\left(\rho_{\text {purge }} / \rho_{f}\right)$ is also calculated to facilitate possible comparisons. The freestream density value is defined as the mean density on the trailing-edge faces, and the density of the purge flow is defined as the one in the cavity plenum. The relation between these two, obtained in the direct computations of the full domain. is shown in Fig. 6a. The results agree with those obtained by Saracoglu et al. [15] and Gorbachova et al. [35]. Additionally, in Fig. 6b, the ratio between the computed base pressure at both sides of the trailing edge $P_{b}$ and the base pressure for a nonblowing configuration $P_{b}^{*}$ is shown as a function of the blowing rate. Pressure values bifurcated for a blowing rate of $17.8 \%$, going back to the symmetric flow state for a blowing rate of $42 \%$ and reaching a maximum value of a $3.6 \%$ of the pressure difference at $28 \%$ of the blowing intensity. The value of the blowing rate for the maximum pressure difference does not match the maximum value for the base pressure, which takes place at a blowing rate of $24 \%$. As will be shown, the reason for this behavior is the appearance of a nonsymmetric global mode that eventually becomes unstable and dominates the flow configuration. With the aim of capturing the instability related with the onset of this bifurcation and identifying the sources of the nonsymmetry, cases in which a blowing rate went from 10 to $45 \%$ are analyzed.

For illustration, the base flow pressure and velocity fields for some of the test cases obtained with mesh M4, including the solution for a nonblowing configuration, are displayed in Figs. 7 and 8 . These figures reveal how the shock waves decrease its intensity for low purge intensities that, combined with the filling effect of the purge jet, increase the pressure of the base region. For blowing rate values higher than $24 \%$, the flow on the dead area starts to evacuate and the pressure at the base region decays, recovering the shock part of its original strength.

The analysis of the solutions obtained with the full mesh allow us to understand the mechanisms of the nonsymmetry. A detailed evolution of the recirculation areas is shown in Fig. 9, in which only negatives velocities are plotted. As the jet is applied at the base region, the recirculation area is pushed downstream, splitting the recirculation region into three main areas: two at the trailing-edge tips and a third one downstream. However, for blowing ratios above the bifurcation point, one of the recirculation bubbles of the trailing edge joins the recirculation area downstream, increasing the pressure on that side and forcing the flow to change its direction in a Coanda effect style. This change alters the wake structure, affecting the shock angles and intensity. Larger blowing rates, however. stabilize the flow, pushing the dead air zones toward the symmetry plane as more flow is entrained into the main jet until the symmetric state is achieved again. The nonsymmetric effect can also be

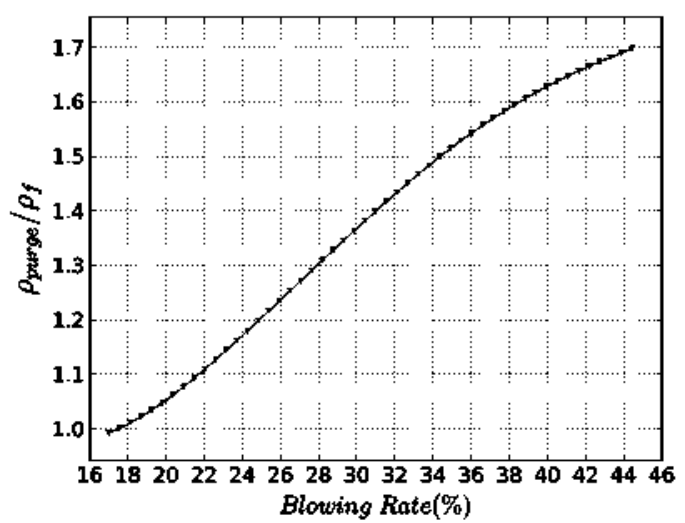

a) Relation between the blowing rate and the density ratio

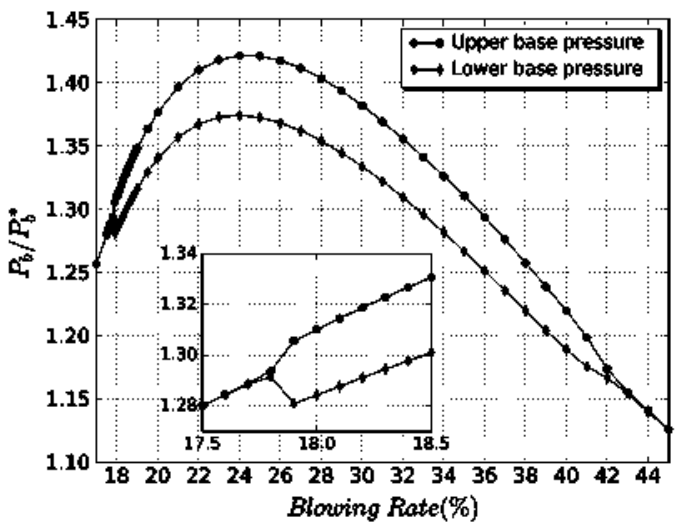

b) Base pressure bifurcation, as a function of the blowing rate

Fig. 6 Density and pressure values measured at the base region. 

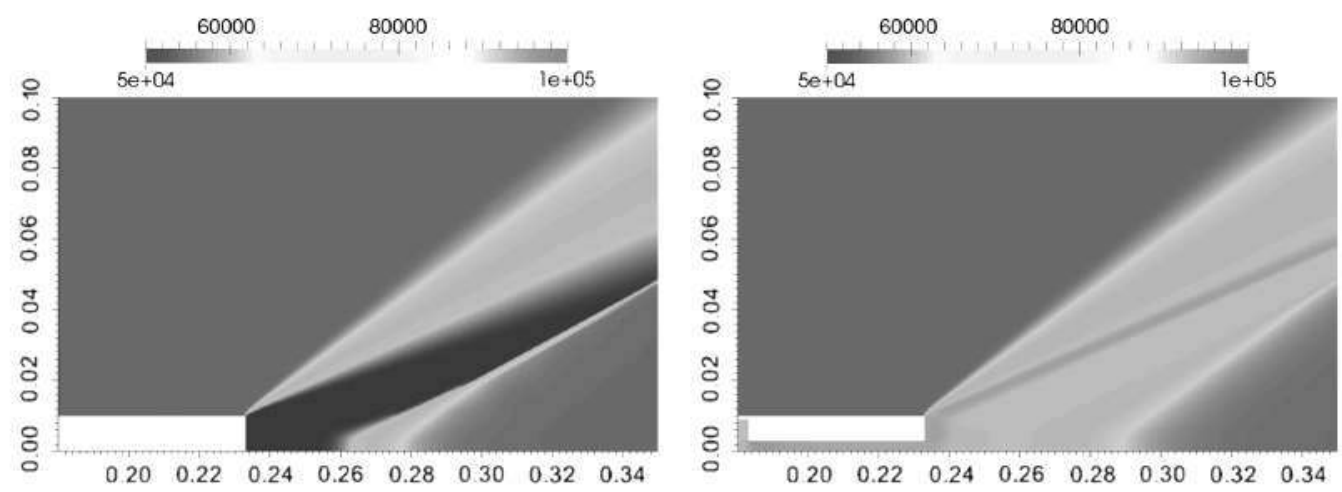

a) No bleeding

b) $17 \%$ blowing rate
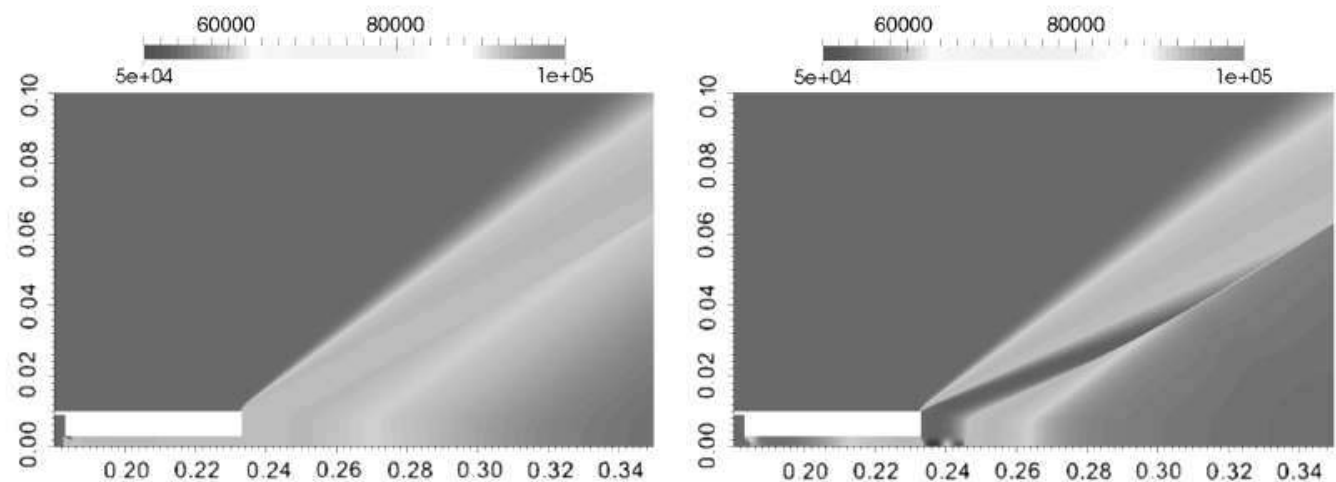

c) $31 \%$ blowing rate

d) $43 \%$ blowing rate

Fig. 7 Base flow computations for different test cases and no bleeding configuration: pressure contours.
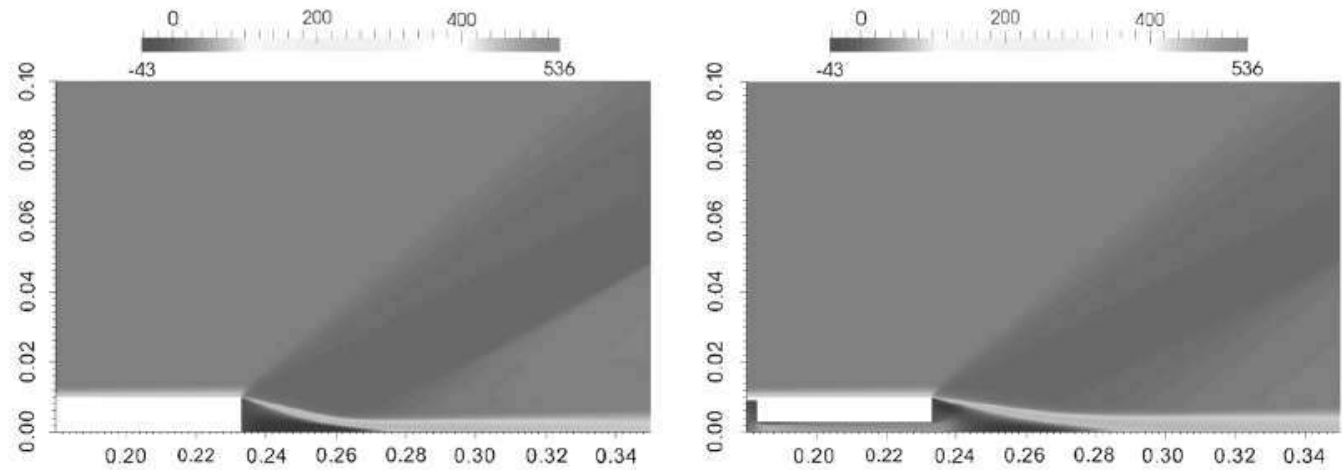

a) No blowing

b) $17 \%$ blowing rate
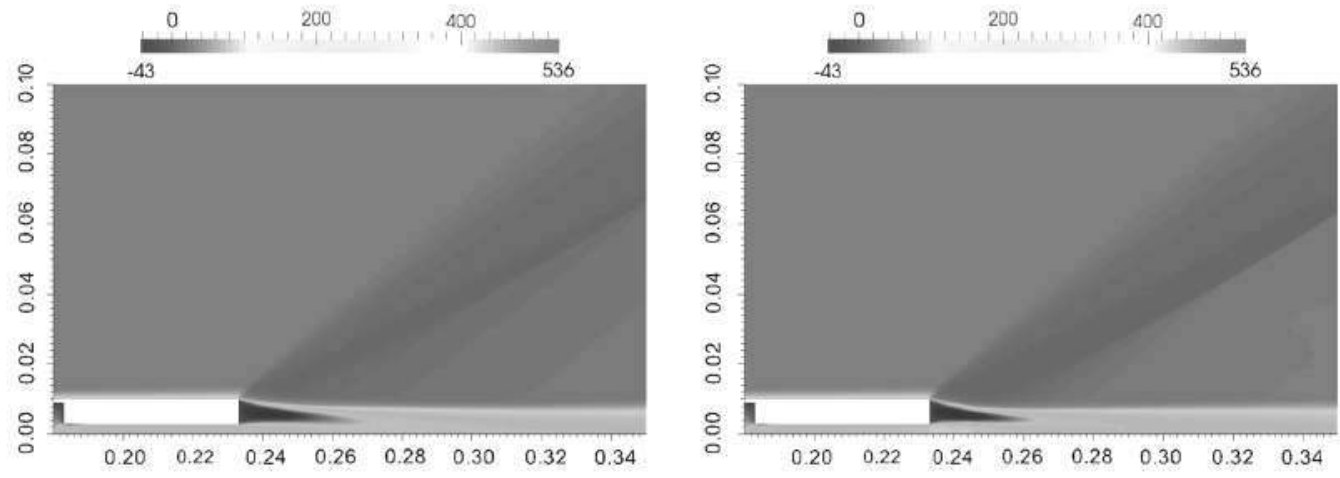

c) $31 \%$ blowing rate

d) $43 \%$ blowing rate

Fig. 8 Base flow computations for different test cases and no bleeding configuration: streamwise velocity contours.

appreciated from shadowgraph contour fields shown in Fig. 10, in which it is easier to identify the weakening of the trailing-edge shock wave and the appearance of a secondary upstream shock only on one side of the domain. The secondary upstream shock has its foot near the region in which the purging flow contacts the trailing-edge shear layers; so, if the flow changes its direction and deflects to the contrary side, the secondary shock will swap side as well. 


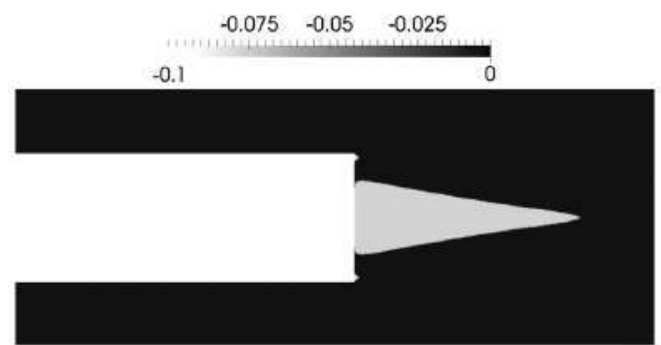

a) No blowing

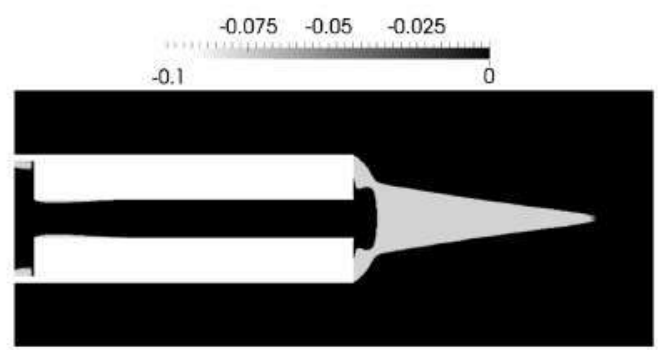

c) $15 \%$ blowing rate

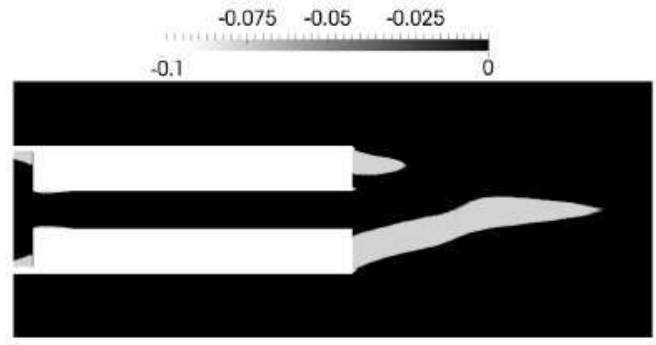

e) $18 \%$ blowing rate

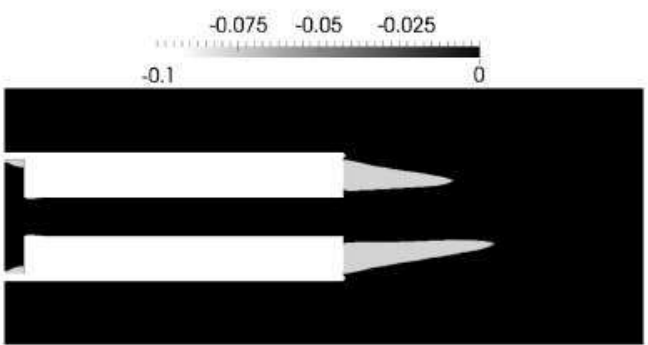

g) $33 \%$ blowing rate

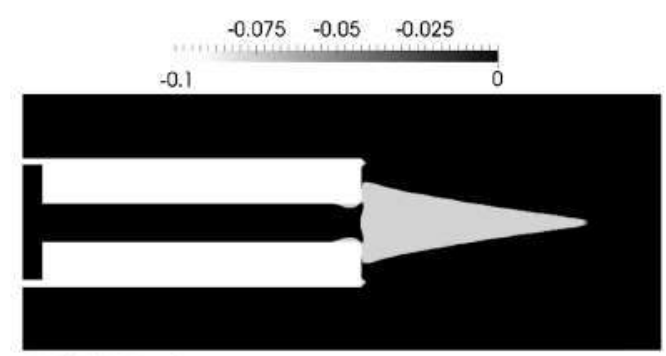

b) $10 \%$ blowing rate

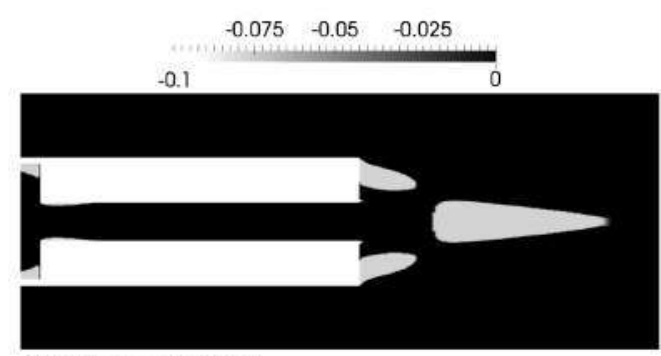

d) $17 \%$ blowing rate

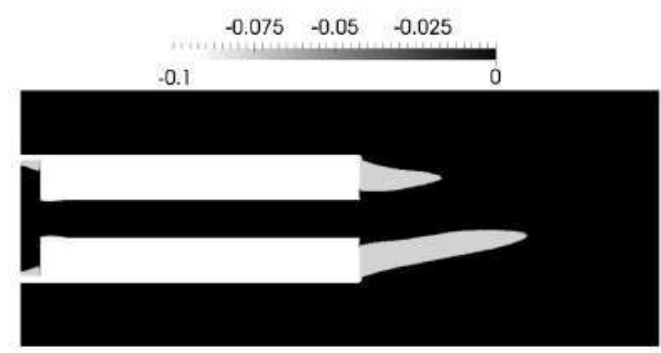

f) $22 \%$ blowing rate

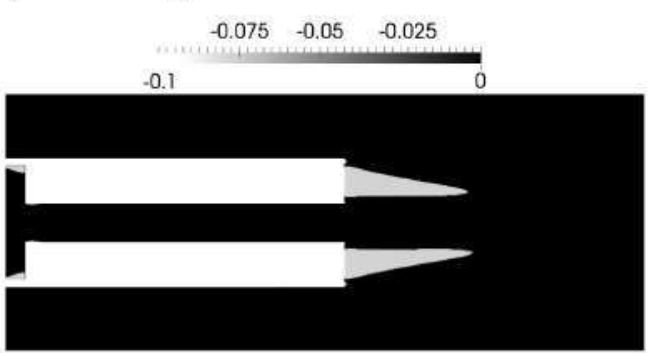

h) $42 \%$ blowing rate

Fig. 9 Dead air regions for different test cases and no bleeding configuration. Only negative velocity values are plotted.

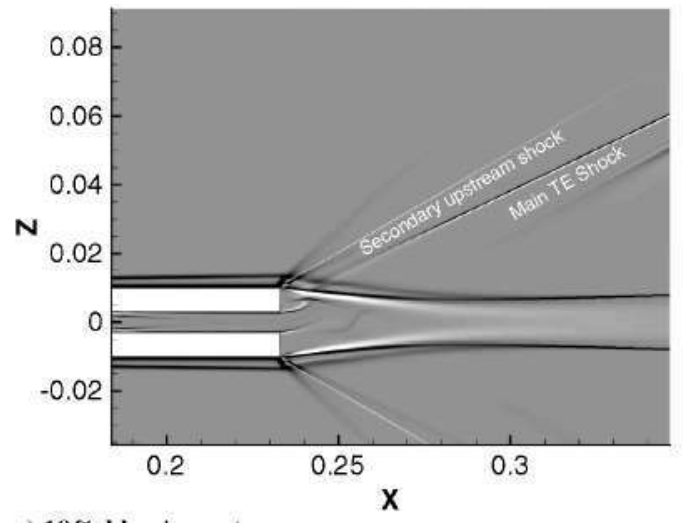

a) $18 \%$ blowing rate

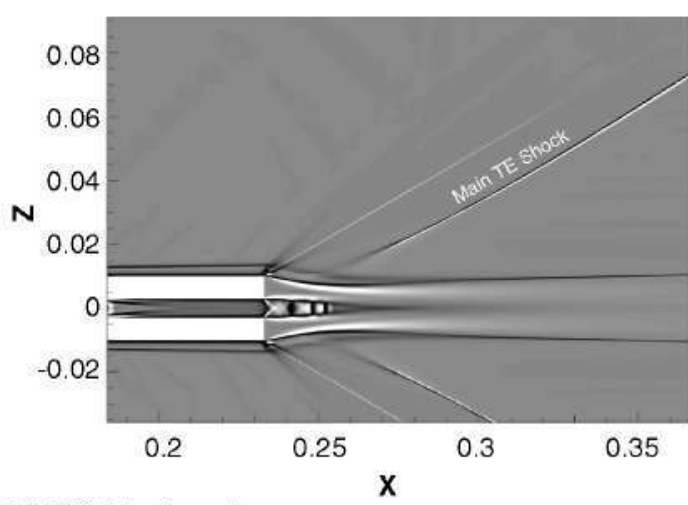

b) $45 \%$ blowing rate

Fig. 10 Shadowgraph contours for a) nonsymmetric and b) symmetric flow configurations (TE, trailing edge).

\section{B. Stability Analysis}

In the work of Saracoglu et al. [15], the pressure measurements were done over a URANS simulation, showing an asymmetrical state that was constant in time, and suggesting that the difference in pressure did not have a periodic oscillation behavior but a steady one.
As shown in the flow topology analysis (Sec IV.A), the cooling flow enters the channel from the cavity area, and suffers a sudden expansion at the base region that is limited on the upper and lower sides by the shear layers. According to the type of perturbation observed on the base flow (Fig. 6a), the geometry configuration, and 


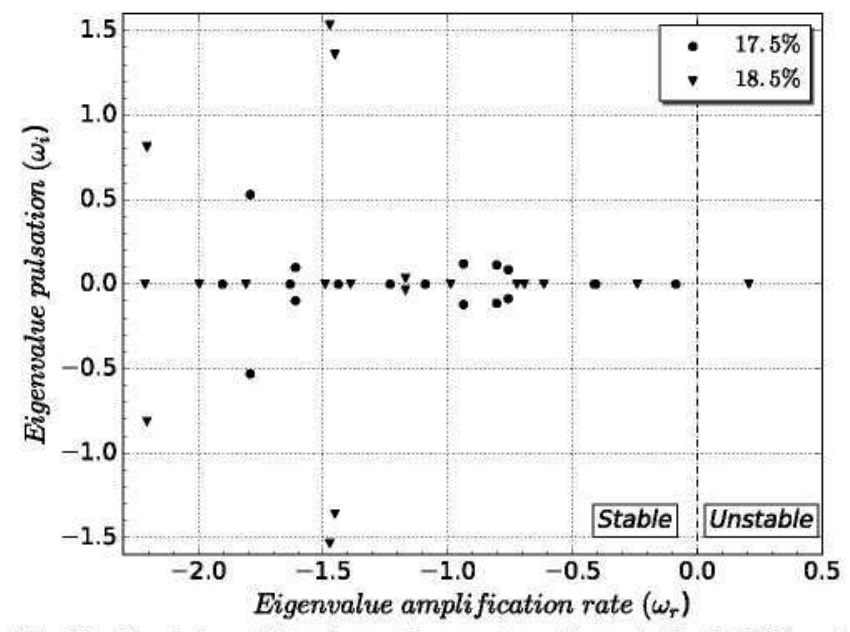

Fig. 11 Evolution of the eigenvalue spectrum for a stable $(17.5 \%)$ and an unstable $(18.5 \%)$ configuration.

the results of Saracoglu et al. [15], it would be expected to find the physical eigenvalues on the imaginary axis having a null pulsation. This kind of instability, known as a pitchfork bifurcation, would lead to steady asymmetric behavior of the recirculation regions at the trailing edge. Therefore, the focus is set on the region of the spectrum close to the origin, and a zero value for the shift parameter is used on the preconditioning of the Jacobian matrix.

As previously explained, the base flows were computed using the half-domain mesh, whereas for the stability analysis, the full-domain mesh was used. All the simulations were performed using the M4 mesh of the convergence study and reduced domain DR3 for the stability analysis. The density, momentum, energy, and turbulent viscosity of the perturbation fields were obtained from this analysis.

For blowing rates below $18 \%$, all the eigenvalues of the spectrum remain on the stable region. When the blowing rate is increased, however, a single antisymmetrical mode becomes unstable and its amplification rate evolves as a function of this parameter (Fig. 11). This mode is directly related to the pressure bifurcation, with its associate eigenvalue crossing the real axis when the purge intensity is above $18.1 \%$ and becoming stable again for a blowing rate of $38 \%$ (Fig. 12). When the eigenvalue evolution is related to the base flow results, it can be observed that, as the base region experiment changes in its flow topology, enlarging the recirculation bubbles of the trailing edge and showing an increment of the pressure drop at the wake (as shown in Figs. 7 and 9), the amplification rate of the unstable eigenvalue reaches its maximum value. After this point, the eigenvalue starts its damping until a blowing rate value of $38 \%$, where it becomes stable again at the same time the base flow recovers its symmetric state.

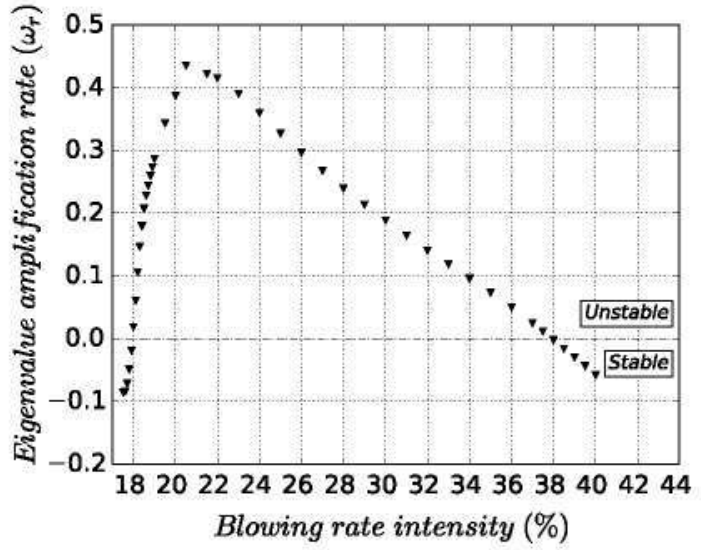

a) Eigenvalue evolution with the blowing rate

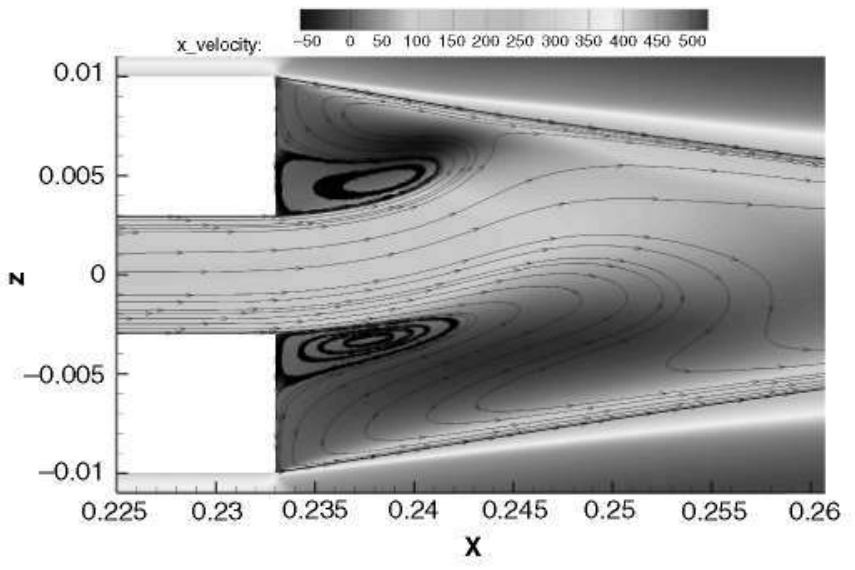

a) Flow solution for a nonsymmetric configuration

Fig. 12 Relation between the global mode and the flow topology.

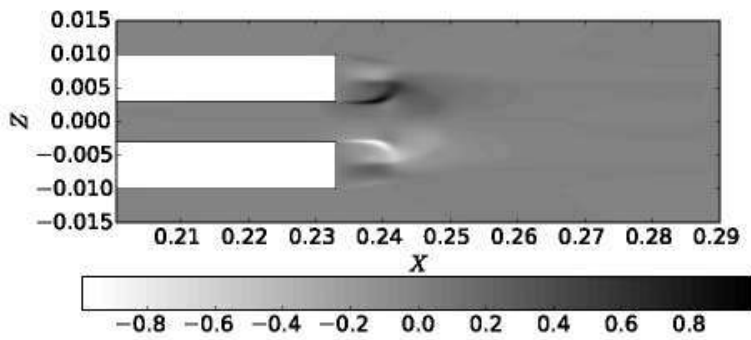

a) $18 \%$ blowing rate

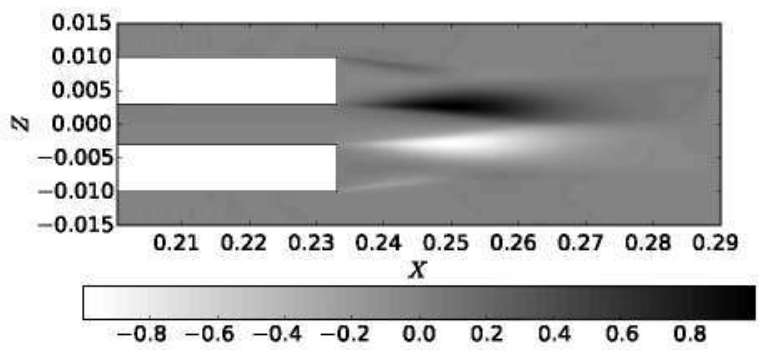

c) $30 \%$ blowing rate

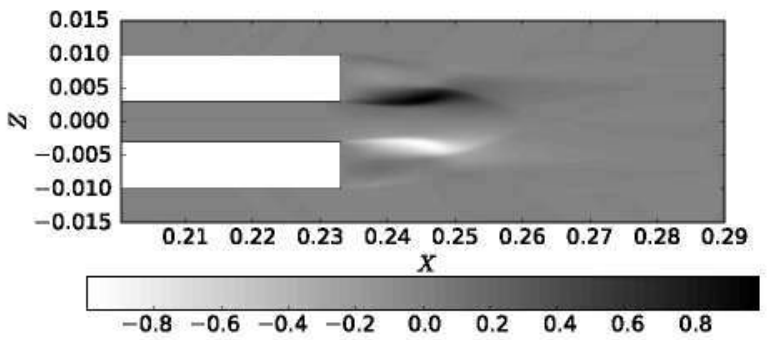

b) $20 \%$ blowing rate

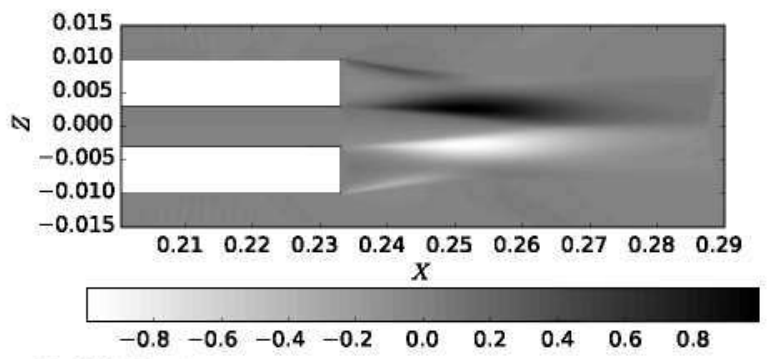

d) $40 \%$ blowing rate

Fig. 13 Evolution of the streamwise component of the eigenmode with the blowing rate. 
The eigenmode structure consists of two symmetrical lobe-shaped regions with antisymmetrical streamwise perturbation components, and it changes its structure as long as the blowing rate increased. which is in consonance with the flow topology of the base flow (Fig. 13). At lower purge intensity cases, the mode appears concentrated at the exit of the purge channel and associated to the weak shear layers that occur in the mixing of the base region and the purge flow. Subsequently, for larger blowing rates, the mode changes its shape, becoming more similar to the structures observed in common channel expansions [36,45], where it adopts the shape of two enlarged lobes bounded by the strong shear layers of the upper and lower tips of the trailing edge.

The behavior of the instability amplification rate is different at low and high blowing intensities. For a weak trailing-edge blowing, the rate of change of the amplification rate of the mode is high, and small variations of blowing rates produce a rapid change of the global mode behavior from stable to unstable; at higher blowing rates, the tendency is smoother and the changes in stability behavior are less abrupt. This fact can also explain the small differences between the RANS results and the stability analysis. Namely, according to RANS computations, the flow remains nonsymmetric until a blowing rate of around $42 \%$, as compared to a value of $38 \%$ for the stability analysis. However, it is normally observed that, when the amplification rate of the instability is not large enough, the flow can remain in an "unstable" situation for a long time, unless a perturbation is introduced in the flow, triggering the new flow configuration [46].

\section{Conclusions}

The phenomenon of the sudden expansion-related instability is replicated in this context for the supersonic blowing trailing-edge problem, which is a simplified version of the flow configuration close to the trailing edge of a turbomachinery turbine blade. The presence of a bifurcation from a symmetric to a nonsymmetric flow configuration as a function of the jet blowing rate had been observed but not explained. A previous analysis of this geometrical model suggested that the bifurcation was generated by a sudden expansion mechanism [15]. This fact is confirmed in this work with the use of the global stability theory.

The presented results link the nonsymmetrical configuration with an unstable antisymmetric eigenmode for a range of blowing rates, matching the start and end points of the pressure bifurcation with small error when compared with Reynolds-averaged Navier-Stokes (RANS) results. Comparing the freestream-to-purge density ratio ( $\rho_{\text {purge }} / \rho_{f}$ ) of RANS solutions and a stability analysis, an error value of $0.5 \%$ is found for low blowing rates, with an error of about $5 \%$ for higher blowing rates. The eigenmode flow structures appear to extend downstream in space as the purge intensity increases, revealing itself as a clear sudden expansion mode for higher blowing rates (having the same structure as those seen in the work of Fani et al. [36]), but is more concentrated at the end of the cooling channel for lower blowing rates. In this range of low blowing rates, the streamwise perturbations of the global mode appear more concentrated near the exit of the channel. The perturbations behave like shear layers constrained by the upper and lower sides of the trailing edge. In this regime, perturbations are more sensitive to changes in the blowing rate.

When compared with a global mode related with a geometrical flow sudden expansion, its similarity in structure seemed clear, despite the absence of viscous walls on the upper and lower sides aft of the expansion that would bound the blow. In the configuration analyzed here, however, the flow was confined due the strong pressure gradients generated by the shear layers present on the trailing edge that limited the base region. This shear layers changed its shape with the blowing rate intensity, modifying in this way the boundaries of the sudden expansion, and thus affecting the eigenmode structure.

As observed, the base pressure magnitude inversely correlated with the trailing-edge shock intensity. The variation of the cooling flow purge initially resulted in an increment of the base pressure, and thus a decrease in the shock strength; for blowing rates higher than $25 \%$, this tendency reversed and the base pressure decayed, resulting in stronger shock waves (Fig. 7). The presence of the instability generates a pressure difference between the upper and lower sides, forcing the flow to deflect to the low pressure side of the trailing edge. This phenomenon appears when the base pressure reaches its maximum value, loosing part of the benefits of the flow injection. Due to the nature of the instability, the cooling flow will not oscillate but keep its deflected direction. However, as both conditions (or branches) of the bifurcation are possible (high pressure on the upper side, and low pressure on the lower side, and viceversa), additional perturbations could generate an abrupt change of the flow direction to the opposite side of the trailing edge, synchronized with a change of the base pressure, that will imply strong load changes on the turbine blades. Despite the apparently low difference between the upper and lower pressure sides (only about 3\%, as shown in Fig. 6), the pressure bifurcation could have a strong effect on the surrounding flow topology and adjacent blades of the turbine. For blowing rates comprised between 18 and $38 \%$, the global instability forced the flow to deflect toward one of the shear layers (Fig. 12b), generating a secondary shock wave upstream of the trailing-edge shock on only one side (Fig. 10). This additional shock system, placed on only one side of the blade, would change to the opposite side when the deflected purge flow changed its direction due to a bifurcation branch change, therefore generating additional and nonsymmetrical loads on the turbine cascade. If this intermittent nonsymmetric shock system was replicated all over the turbine blades, considering independent changes and interactions with upstream and downstream stages of the turbine, the consequences in the aerodynamic loads could be very important. To avoid these risks, one should increase the blowing rate up to a state of no variation (symmetry), implying an important increment on the base pressure losses and the intensity of the trailing-edge shock wave system. The non-time-dependent nature of the instability would made it difficult to control by frequency-modulated systems (such as those proposed by Saracoglu et al. [10]), but it might be suitable for passive control or upstream flow modulation. The possibility of controlling the pressure bifurcation and avoiding the aforementioned problems would allow the exploitation of lower blowing rates for base region modulation without undesired consequences.

Understanding the mechanisms of the nonsymmetric flow configurations present in a supersonic trailing edge, using a simplified model as the one described in this work, is the first step in preventing unwanted loads on turbine blades by the global stability analysis. The methodology explained here can be applied without changes to more realistic cases in which the pressure gradient between both sides of the airfoil can play an important role in damping/exciting the sudden expansion mode. A closer approximation to the problem could allow damping of its sources (the unstable eigenmode) and even development of optimization techniques with direct feedback from the eigenvalue analysis.

\section{Acknowledgments}

This research was carried out under the project Stability and Sensitivity Methods for Industial Design, which received funding from the European Union Horizon 2020 research and innovation program under Marie Skłodowska-Curie grant agreement no. 675008 . The authors thankfully acknowledge the computer resources and technical assistance provided by the Centro de Supercomputación y Visualización de Madrid (CeSViMa).

\section{References}

[1] Nash, J. F., "A Review of Research on Two-Dimensional Base Flow". Aeronautical Research Council R\&M No. 3323, Great Britain, 1963. pp. 1-25.

[2] Nash, J. F., "A Discussion of Two-Dimensional Turbulent Base Flows." Aeronautical Research Council R\&M No. 3468, Great Britain, 1967. pp. 1-46.

[3] Nash, J. F., Quincey, V. G., and Callinan, J., "Experiments on TwoDimensional Base Flow at Subsonic and Transonic Speeds." Aeronautical Research Council R\&M No. 3427, Great Britain, 1966. pp. 1-34. 
[4] Hama, F. R., "Experimental Studies on the Lip Shock," AlAA Journal. Vol. 6. No. 2, 1968, pp. 212-219. doi: $10.2514 / 3.4480$

[5] Sieverding. C. H.. Stanislas, M.. and Snoek. J., "The Base Pressure Problem in Transonic Turbine Cascades," Jounal of Engineering for Power, Vol. 102, No. 3, 1980, pp. 711-718. doi: $10.1115 / 1.3230330$

[6] Denton. J. D., and Xu. L., "The Trailing Edge Loss of Transonic Turbine Blades," Journal of Turbonachinery, Vol. 112, No. 2, 1990, pp. 277-285. doi: $10.1115 / 1.2927648$

[7] Herin. J. L., and Dutton. J. C., "Supersonic Base Flow Experiments in the Near Wake of a Cylindrical Afterbody," AIAA Journal, Vol. 32, No. 1. 1994, pp. 77-83. doi: $10.2514 / 3.11953$

[8] Sandberg, R. D., "Numerical Investigation of Turbulent Supersonic Axisymmetric Wakes,"Joumal of Fluid Mechanics, Vol. 702. July 2012. pp. $488-520$. doi: $10.1017 / \mathrm{ffm} .2012 .201$

[9] Sandherg. R. D.. and Fasel. H. F., "Numerical Investigation of Transitional Supersonic Axisymmetric Wakes." Jowmal of Fluid Mechunics, Vol. 563. Sept. 2006. pp. 1-41. doi: $10.1017 / \mathrm{S} 0022112006000899$

[10] Saracoglu. B. H.. Paniagua, G., Salvadori. S., Tomasoni. F., Duni, S.. Yasa, T., and Miranda. A.." "Trailing Edge Shock Modulation by Pulsating Coolant Ejection," Applied Thermal Engineering. Vol. 48, April 2012. pp. 1-10.

doi: 10.1016/j.applthermaleng.2012.04.036

[11] Raffel, M., and Kost, F. "Investigation of Aerodynamic Effects of Coolant Ejection at the Trailing Edge of a Turbine Blade Model by PIV and Pressure Measurements." Experiments in Fluids, Vol. 24, Nos. 5-6, 1998, pp. 447-461. doi: $10.1007 / \mathrm{s} 003480050194$

[12] Kost.F. H., and Holmes, A. T. "Aerodynamic Effect of Coolant Ejection in the Rear Part of Transonic Rotor Blades," AGARD Heut Transfer and Cooling in Gas Turbines 12 p, Rolls Royce Ltd. SEE N86-29823 21-07. England. Sept. 1985.

[13] Bohn, D. E., Becker, V. J., Behnke, K. D., and Bonhoff, B. F., "Experimental and Numerical Investigations of the Aerodynamical Effects of Coolant Injection Through the Trailing Edge of a Guide Vane," Proceedings of the ASME International Gas Turbine and Aeroengine Congress and Exposition, American Soc. of Mechanical Engineers Paper 95-GT-26, Fairfield, NJ. 1995. doi: 10.1115/95-GT-026

[14] Wang, Y., and Zhao, L., "Investigation on the Effect of Trailing Edge Ejection on a Turbine Cascade," Applied Mathematical Modelling. Vol. 37. No. 9, 2013, pp. 6254-6265. doi: $10.1016 / j$.apm. 2013.01 .023

[15] Saracoglu. B. H.. Paniagua, G., Sanchez, J.. and Rambaud, P.. "Effects of Blunt Trailing Edge Flow Discharge in Supersonic Regime." Computers and Fluids, Vol. 88, Sept. 2009, pp. 200-209. doi: $10.1016 /$ j.compfluid. 2013,09.013

[16] Iorio, M. C., González. L. M., and Ferrer, E.., "Direct and Adjoint Global Stability Analysis of Turbulent Transonic Flows over a NACA0012 Profile," International Journal for Numerical Methods in Fluids. Vol. 76. No. 3, 2014, pp. 147-168. doi: $10.1002 / 1$ ld .v76.3

[17] Iorio. M. C., González. L. M., and Martinez-Cava, A.. "Global Stability Analysis of a Compressible Turbulent Flow Around a High-Lift Configuration," AIAA Journal, Vol. 54, No. 2, 2016, pp. 373-385. doi: $10.2514 / 1 . J 054211$

[18] Sartor, F., Mettot, C., Bur. R., and Sipp. D.. "Unsteadiness in Transonic Shock-Wave/Boundary-Layer Interactions: Experimental Investigation and Global Stability Analysis." Joumal of Fltid Mechanics. Vol. 781. Oct. 2015, pp. 550-577. doi: $10.1017 / \mathrm{jfm} .2015 .510$

[19] Barkley, D., "Linear Analysis of the Cylinder Wake Mean Flow." Europhysics Letters (EPL). Vol. 75. No. 5, 2006, pp. 750-756. doi: $10.1209 / \mathrm{epl} / \mathrm{i} 2006-10168-7$

[20] Oberleithner, K., Rukes. L., and Soria. J.. "Mean Flow Stability Analysis of Oscillating Jet Experiments." Joumal of Fluid Mechanics. Vol. 757, 2014. pp. 1-32. doi: $10.1017 / \mathrm{jfm} .2014 .472$

[21] Sipp, D., and Lebedev, A., "Global Stability of Base and Mean Flows: A General Approach and Its Applications to Cylinder and Open Cavity Flows," Journal of Fludd Mechanics, Vol. 593, Dec. 2007, pp. 333-358. doi: $10.1017 / \mathrm{S} 0022112007008907$

[22] Schmid. P. J., "Dynamic Mode Decomposition of Numerical and Experimental Data," Joumal of Fluid Mechanics, Vol. 656, Aug. 2010, pp. 5-28.
[23] Grilli, M.. Schmid. P. J.. Hickel, S., and Adams. N. A., "Analysis of Unsteady Behaviour in Shockwave Tutbulent Boundary Layer Interaction," Joumal of Fluid Mechanics, Vol. 700, No. 700, 2012, pp. 16-28. doi: $10.1017 / \mathrm{jtm} .2012 .37$.

[24] Lashgari, I., Tammisola, O., Citro, V., Juniper, M. P., and Brandt, L.. "The Planar X-Junction Flow: Stability Analysis And Control," Journal of Fluid Mechanics. Vol. 753. Aug. 2014. pp. 1-28. doi: $10.1017 / \mathrm{fm} .2014 .364$

[25] Wilcox. D. C.. "Fonmulation of the k-w Turbulence Model Revisited," AlAA Journal. Vol. 46, No. 11.2008, pp. 2823-2838. doi: $10.2514 / 1.36541$

[26] Schwambom. D., Gerhold, T.. and Heinnich, R.. "The DLR Tau-Code: Recent Applications in Research and Industry." Proceedings of the European Conference on Computational Fluid Dvnanics, Edmond aan Zee, Delft Unjv, of Technology. Delft, The Netherlands, 2006.

[27] Liou, M.-S.. and Steffen, C. J.. "A New Flux Splitting Scheme," Journal of Computational Physics, Vol. 107, No. 1, 1993, pp. 23-39. doi: 10.1006 j jcph.1993.1122

[28] Anderson. J. D., and Wendt, J., Computational Fluid Dynamics, Vol. 206, Springer, New York, 1995.

[29] Chomaz, J.-M.. "Global Instabilities in Spatially Developing Flows: Non-Nonnality And Nonlinearity." Ammual Review of Fltid Mechanics, Vol. 37, No. 1, 2005, pp. 357-392. doi: $10.1146 / a n n u r e v$ fluid 37.061903 .175810

[30] Theofilis. V., "Global Linear Instability." Anmual Review: of Fiud Mechunics, Vol. 43, No. 1, 2011,pp. 319-352. doi: 10.1 146/annurev-fluid-122109-160705

[31] Luchini, P.. and Bottaro, A., "Adjoint Equations in Stability Analysis," Annual Review of Fluid Mechanics, Vol. 46. No. 1, 2014, pp. 493-517. doi: 10.1 146/annurev-fluid-010313-141253

[32] Taira, K., Brunton. S. L., Dawson. S. T. M., Rowley. C. W., Colonius. T., McKeon, B. J., Schmidt, O. T. Gordeyev, S., Theofilis, V, and Ukeiley, L. S., "Modal Analysis of Fluid Flows: An Overview" AIAA Joumal. Vol. 55, No. 12.2017, pp. 4013-4041. doi: $10.2514 / 1 . J 056060$

[33] Meseguer-Garrido, F., De Vicente, J., Valero, E., and Theofilis, V., "On Linear Instability Mechanisms in Incompressible Open Cavity Flow," Joumal of Fluid Mechanics. Vol. 752, No. 2014, Aug. 2014, pp. 219-236. doi: $10.1017 / \mathrm{jfm} .2014 .253$

[34] González, L. M.. Ahmed. M.. Kühnen. J.. Kuhlmann, H... and Theofilis, V., "Three-Dimensional Flow Instability in a Lid-Driven Isosceles Triangular Cavity." Journal of Fluid Mechanics. Vol. 675, May 2011. p19. 369-396. doi: $10.1017 / \mathrm{S} 002211201100022 \mathrm{X}$

[35] Gorbachova, Y., Valero, E., Paniagua, G., Martinez-Cava, A., and Saracoglu, B. H., "Study of the Attainable Flow Topologies in a Supersonic Blunt Trailing Edge at Various Blowing Ratios." 5tth AlAA Aerospace Sciences Meefing and ALAA SciTech Forum. AIAA Paper 2016-0909, 2016. doi: $10.2514 / 6.2016-0909$.

[36] Fani, A., Camarri, S., and Salvetti, M. V., "Stability Analysis and Control of the Flow in a Symmetric Channel with a Sudden Expansion," Plusics of Fluids. Vol. 24. No. 8. 2012, Paper 084102. doi: $10.1063 / 1.4745190$.

[37] Fraysse, F., Valero, E., and Rubio, G., "Quasi-A Priori Tnuncation Error Estimation and Higher Order Extrapolation for Non-Linear Partial Differential Equations," Journal of Computational Physics, Vol. 253, Nov. 2013, pp. 389-404. doi: 10.1016/j.jcp.2013.07.018

[38] Lehoucq, R. B., Sorensen. D. C., and Yang, C., "ARPACK Users" Guide: Solution of Large Scale Eigenvalue Problems by Implicitly Restarted Amoldi Methods," Sofnvare, Environments and Tools, Soc. for Industrial and Applied Mathematics (SLAM), Philadelphia, PA, 1998. doi: $10.1137 / 1.9780898719628$

[39] Saad. Y.. Numerical Methods for Large Eigemalue Problems. Soc. for Industrial and Applied Mathematics. Philadelphia, 2011. doi: $10.1137 / 1.9781611970739$

[40] De Vicente, J.. Rodríguez, D., Theofilis, V, and Valero. E.. "Stability Analysis in Spanwise-Periodic Double-Sided Lid-Driven Cavity Flows with Complex Cross-Sectional Protiles." Computers and Fluids. Vol. 43, No. 1, 2011 . pp. 143-153. doi: 10.1016/j.comptluid.2010.09.033

[41] Ferrer, E., De Vicente, J., and Valero, E., "Low Cost 3D Global Instability Analysis and Flow Sensitivity Based on Dynamic Mode Decomposition and High-Order Numerical Tools," Intemational Joumal for Numerical Methods in Fluids, Vol. 76, No. 3, 2014, pp. 169-184. doi: $10.1002 / \mathrm{fld} . \mathrm{v} 76.3$ 
[42] Browne. O. M. F., Rubio. G., Ferter, E.. and Valero. E.. "Sensitivity Analysis to Unsteady Perturbations of Complex Flows: A Discrete Approach." International Journal for Numerical Methods in Fluids, Vol. 76, No. 12. 2014, pp. 1088-1110. doi: $10.1002 /$ fld.v76.12

[43] Amestoy, P. R., Duff, I. S., L'Excellent, J.-Y., and Koster, J., "A Fully Asynchronous Multifrontal Solver Using Distributed Dynamic Scheduling." SIAM Joumal on Matrix Analysis and Applications. Vol. 23, No. 1, 2001, pp. 15-41. doi: $10.1137 / \mathrm{S} 0895479899358194$

[44] Sanvido. S., Garicano-Mena, J., De Vicente, J., and Valero. E., "Critical Assessment of Domain Reduction Strategies for Global Stability Analysis." Communications in Computational Physics (submitted for publication?.
[45] Mizushima. J.. and Shiotani, Y.. "Transitions and Instabilities of Flow in a Symmetric Channel with a Suddenly Expanded and Contracted Part," Joumul of Fluid Mechanics, Vol. 434, May 2001, pp. 355-369.

doi: $10.1017 / S 0022112001003743$

[46] Valero, E., Ferrer, E., and de Vicente, J., "Numerical Methods for Direct Numerical Simulation and Stability Analysis." Progress in Flow Instability Analysis and Laminar-Turbulem Transition Modeling, VKI LS 2014-07, edited by E. Valero, and F. Pinna. von Kánmán Inst. for Fluid Dynamics, Sint-Genesius-Rode. Belgium, 2014.

C. W. Rowley Associate Editor 